\title{
A CONSERVATION RESULT CONCERNING BOUNDED THEORIES AND THE COLLECTION AXIOM
}

\author{
SAMUEL R. BUSS
}

\begin{abstract}
We present two proofs, one proof-theoretic and one model-theoretic, showing that adding the $B \Sigma_{1}^{0}$-collection axioms to any bounded first-order theory $R$ of arithmetic yields an extension which is $\forall \Sigma_{1}^{0}$-conservative over $R$.
\end{abstract}

Preliminaries. A theory of arithmetic $R$ contains the nonlogic symbols $0, S,+, \cdot$, and $\leqslant . R$ may contain further nonlogical symbols; in particular $S_{2}$ is a theory of arithmetic [1]. We shall say that $R$ is sufficient if and only if $R$ proves

(a) $\leqslant$ is a linear ordering.

(b) For every term $t(\vec{x})$, there is a term $\sigma_{t}$ such that

$$
R \vdash x_{1} \leqslant y_{1} \wedge \cdots \wedge x_{k} \leqslant y_{k} \rightarrow t(\vec{x}) \leqslant \sigma_{t}(\vec{y}) .
$$

Of course, the usual bounded theories of arithmetic, for example $I \Delta_{0}$ or $S_{2}^{1}$, are sufficient. Indeed letting $\sigma_{t}$ be $t$ suffices for these theories. Although Theorem 1 below holds for second order bounded theories of arithmetic such as $U_{2}^{i}$ and $V_{2}^{i}$, we shall only discuss first order theories in this paper. From now on, $R$ is presesumed to be a first order theory.

The syntax of first order logic is enlarged to include bounded quantifiers of the forms $(\forall x \leqslant t)$ and $(\exists x \leqslant t)$, where $t$ is any term not containing $x$. In [1] it is shown how Gentzen's sequent calculus $L K$ may be enlarged to incorporate bounded quantifiers. A formula is bounded if and only if it contains no unbounded (i.e., usual) quantifiers. A theory $R$ of arithmetic is bounded if and only if $R$ is axiomatized by a set of bounded formulae.

The class of $\Sigma_{1}^{0}$-formulae is defined to contain those formulae in which each unbounded quantifier is either existential and in the scope of an even number of negations, or universal and in the scope of an odd number of negations. Note that our definition of $\Sigma_{1}^{0}$ is slightly broader than the set of $\Sigma_{1}$ formulae defined by Paris and Kirby [4]. The $B \Sigma_{1}^{0}$-collection axioms are

$$
(\forall x \leqslant a)(\exists y) A(x, y) \rightarrow(\exists z)(\forall x \leqslant a)(\exists y \leqslant z) A(x, y),
$$

where $A$ is any $\Sigma_{1}^{0}$-formula [4]. Note that $A$ may contain additional free variables as parameters. The $B \Sigma_{1}^{0}$-collection axioms are equivalent to the $B \Sigma_{1}^{0}$-collection axioms of Paris and Kirby [4] since the $B \Sigma_{1}^{0}$-collection can prove that every $\Sigma_{1}^{0}$ formula is

Received by the editors November 18, 1985 and, in revised form, February 21, 1986, 1980 Mathematics Subject Classification (1985 Revision). Primary 03C30, 03B99.

Key words and phrases. Bounded arithmetic, collection axioms, cut elimination, resplendency. 
equivalent to a $\Sigma_{1}$-formula. The class $\forall \Sigma_{1}^{0}$ of formulae is the set of sentences which are universal closures of $\Sigma_{1}^{0}$-formulae.

The object of this paper is to prove

THEOREM 1. Let $R$ be a bounded, sufficient theory of arithmetic. Then $R+B \Sigma_{1}^{0}$ is $\forall \Sigma_{1}^{0}$-conservative over $R$ (in other words, every $\forall \Sigma_{1}^{0}$-consequence of $R+B \Sigma_{1}^{0}$ is a theorem of $R$ ).

It has been known for some time that $I \Delta_{0}+B \Sigma_{1}^{0}$ is $\Pi_{2}^{0}$-conservative over $I \Delta_{0}$. However, the proof of this by Paris [3] does not extend readily to prove Theorem 1. This author first discovered the proof-theoretic proof of Theorem 1 after Alex Wilkie brought Paris' theorem to his attention. Later, a result of J. P. Ressayre [5] prompted the author's discovery of a model-theoretic proof based on resplendency.

Both proofs are presented below and they are independent and self-contained; so the reader should feel free to read only the one which he or she prefers.

The proof-theoretic proof. We shall work with the sequent calculus $L K B$, which is Gentzen's system $L K$ enlarged to include bounded quantifiers (see [1, Chapter 4]). In addition to the inference of $L K B$ we allow inferences given by the $B \Sigma_{1}^{0}$-collection rule:

$$
\frac{\Gamma \rightarrow(\forall x \leqslant t)(\exists y) A(x, y), \Delta}{\Gamma \rightarrow(\exists z)(\forall x \leqslant t)(\exists y \leqslant z) A(x, y), \Delta}
$$

where $\Gamma$ and $\Delta$ denote arbitrary cedents of formulae and $A$ must be a $\Sigma_{1}^{0}$-formula.

LEMMA 2. The $B \Sigma_{1}^{0}$-collection axion and the $B \Sigma_{1}^{0}$-collection rule are equivalent.

Proof. This is obvious.

A proof $P$ of the sequent calculus $L K B$ plus $B \Sigma_{1}^{0}$-collection is a tree of sequents $\Gamma \rightarrow \Delta$ where $\Gamma$ and $\Delta$ are lists of formulae. Each node in the proof tree must be a valid inference. The lowest sequent, or root, of $P$ is called the endsequent or the conclusion of $P$. The leaves, or highest sequents, of $P$ are the initial sequents of $P$. When $A$ is an occurrence of a formula in an upper sequent of an inference of $P$, the successor of $A$ is defined to be the formula in the lower sequent of the same inference which corresponds to $A$. Except when $A$ is the principal formula of a cut (modus ponens) inference $A$ always has a unique successor. If $A_{i+1}$ is a successor of $A_{i}$ for all $i<k$, then $A_{k}$ is defined to be a descendant of $A_{0}$. If in addition $A_{0}$ and $A_{k}$ are occurrences of the same formula, then $A_{k}$ is a direct descendant of $A_{0}$.

We modify the definition [1] of a free cut somewhat to account for the new collection rules.

DEFinition. A cut is free if and only if neither of the following hold:

(1) one of the principal formulae of the cut is a direct descendant of a formula in an initial sequent (i.e., in an axiom),

(2) one of the principal formulae of the cut is a direct descendant of the principal formula of a $B \Sigma_{1}^{0}$-collection inference. 
LEMMA 3. Let $R$ be any first order theory. The free cut elimination theorem holds for $R+B \Sigma_{1}^{0}$. Namely, if $P$ is an $\left(R+B \Sigma_{1}^{0}\right)$-proof, then there is a free-cut free $\left(R+B \Sigma_{1}^{0}\right)$-proof $P^{*}$ with the same conclusion as $P$ so that the principal formulae of collection inference in $P^{*}$ are instances of the principal formulae of collection inferences of $P$.

The proof of Lemma 3 follows the usual proof of the cut-elimination theorem (see Takeuti [6]).

Lemma 4. Let $R$ be a bounded, sufficient theory of arithmetic and suppose $A \in \Sigma_{1}^{0}$ and $R \vdash A$. Further suppose $A$ contains a subformula of the form $(\exists x) B$. That is to say, $A=C((\exists x) B)$, where $C(\alpha)$ contains only a single instance of the second order variable $\alpha$. Then there is a term $t$ such that

$$
R \vdash C((\exists x \leqslant t) B) .
$$

Proof (OUTLINe). This is a corollary to a theorem of Parikh [2]. For our purposes, it is useful to see that Lemma 4 can be proved by the method of proof of Theorem 4.11 of [1]. This proof consists of three parts. First, by cut elimination, there is a free-cut free $R$-proof of $A$. Second, it can be shown that in this free-cut free proof all of the free variables can be explicitly bounded; that is to say, for each free variable $b_{i}$ there is a term $u_{i}$ such that $b_{i}$ is restricted to be less than $u_{i}$ and further the only variables of $u_{i}$ are the free variables of $A$. Finally, it is easy to see that whenever an unbounded existential quantifier is introduced, it can be explicitly bounded by a term involving only the free variables of $A$. The reader should refer to [1] for complete details. (Actually, the proof is easier here than for Theorem 4.11 of [1] since there are no induction inferences in $R$.) Note that the proof depends strongly on $R$ being both bounded and sufficient.

Lemma 4 can be strengthened to apply to theories with $B \Sigma_{1}^{0}$-collection: this is the content of Lemma 5.

Lemma 5. Let $R$ be a bounded, sufficient theory of arithmetic and suppose $A \in \Sigma_{1}^{0}$ and $R+B \Sigma_{1}^{0} \vdash A$. Further suppose $A=C((\exists x) B)$, where $C(\alpha)$ contains only $a$ single instance of $\alpha$. In addition assume that there is a free-cut free $\left(R+B \Sigma_{1}^{0}\right)$-proof a $P$ of $A$ so that the occurrence of $A$ in the endsequent of $P$ is not a descendant of the principal formula of any collection inference in $P$. Then there is a term $t$ and a free-cut free $\left(R+B \Sigma_{1}^{0}\right)$-proof $P^{*}$ of $C((\exists x \leqslant t) B)$ such that $P^{*}$ and $P$ have the same number of collection inferences.

Proof. Since $A$ is not a descendant of the principal formula of a collection inference, the construction used in the proof of Lemma 4 still applies.

If $R$ is any theory and $P$ is a $\left(R+B \Sigma_{1}^{0}\right)$-proof, we say that $P$ is good if and only if for every cut inference in $P$ either its principal formula is bounded or one of its principal formulae is a direct descendant of the principal formula of a $B \Sigma_{1}^{0}$-collection inference. Since $R$ is a bounded theory, every direct descendant of a formula in an initial sequent is bounded; hence every free-cut free proof is good. 
We define some further syntactic properties of a sequent calculus proof $P$. An inference branch of $P$ is a sequence of inferences $I_{1}, \ldots, I_{k}$ such that every upper sequent of $I_{1}$ is an initial sequent of $P$, the lower sequent of $I_{k}$ is the endsequent of $P$, and for $1 \leqslant j<k$, the lower sequent of $I_{j}$ is an upper sequent of $I_{j+1}$. If $B$ is an inference branch of $P$ and $I$ is an inference of $P$, then $I$ is to the left of $B$ if and only if $I$ is not in $B$ and $I$ is on the left side of $B$ in the proof tree $P$. It is important for the definition that upper sequents of inferences are always ordered in the usual fashion (as in [1] or Takeuti [6]). If $I$ and $J$ are inferences, then $I$ is to the left of $J$ if and only if $I$ is to the left of every inference branch containing $J$.

We are now ready to prove the main lemma for Theorem 1.

LEMMA 6. Let $R$ be any bounded and sufficient theory of arithmetic. Suppose $P$ is a good $\left(R+B \Sigma_{1}^{0}\right)$-proof of a $\Sigma_{1}^{0}$-formula $A$. Then $R \vdash A$.

Proof. The proof is by induction on the number $c$ of uses of the $B \Sigma_{1}^{0}$-collection rule in $P$. For $c=0$, this is trivial. So suppose $c \geqslant 1$. Let $I$ be the unique collection inference of $P$ such that no other collection inference is above or to the left of $I$. So $I$ is of the form

$$
\frac{\Gamma \rightarrow(\forall x \leqslant t)(\exists y) C(x, y), \Delta}{\Gamma \rightarrow(\exists z)(\forall x \leqslant t)(\exists y \leqslant z) C(x, y), \Delta} .
$$

Let $Q$ be the subproof of $P$ which has $I$ as its root.

We claim that every formula in $\Gamma$ is an $\Pi_{1}^{0}$-formula and every formula in $\Delta$ is a $\Sigma_{1}^{0}$-formula. If not, let $B \notin \Sigma_{1}^{0}$ and $B \in \Delta$, or $B \notin \Pi_{1}^{0}$ and $B \in \Gamma$. Since no descendant of $B$ can appear in the endsequent of $P$ and no descendant of $B$ can be the principal formula of a $B \Sigma_{1}^{0}$-collection inference and since $P$ is good, it must be the case that some descendant $E$ of $B$ is a $\Sigma_{1}^{0}$-formula in the antecedent of a sequent and is removed by a cut inference and the formula against which $E$ is cut must be a direct descendant of the principal formula of a collection inference. But this is impossible since there is no collection inference to the left of $I$ and the claim is established.

Since $Q$ is an $R$-proof except for its last inference, it now follows by Lemma 4 that there is a term $s$ so that $R$ proves

$$
\Gamma \rightarrow(\forall x \leqslant t)(\exists y \leqslant s(x)) C(x, y), \Delta .
$$

By the sufficiency of $R$ and by Lemma 3, there is a good $R$-proof $Q^{*}$ which has final inference:

$$
\frac{\Gamma \rightarrow(\forall x \leqslant t)\left(\exists y \leqslant \sigma_{s}(t)\right) C(x, y), \Delta}{\Gamma \rightarrow(\exists x)(\forall x \leqslant t)(\exists y \leqslant z) C(x, y), \Delta} .
$$

Replace the subproof $Q$ of $P$ by $Q^{*}$ to form the proof $P^{*}$. If $P^{*}$ is good, we are done since $P^{*}$ has one less collection inference than $P$. So suppose $P^{*}$ is not good. Then there is a subproof of $P^{*}$ of the form

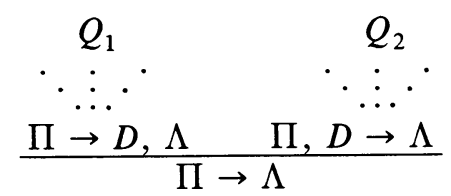


where $Q^{*}$ is a subproof of $Q_{1}, D \in \Sigma_{1}^{0}$, and $D$ is a direct descendant of the principal formula of the last inference of $Q^{*}$. Since $Q_{1}$ has fewer than $c B \Sigma_{1}^{0}$-collection inferences and by the induction hypothesis, there is an $R$-proof $Q_{1}^{*}$ of $\Pi \rightarrow D$, $\Lambda$.

Let the unbounded quantifiers of $D$ be $\left(Q_{1}, x_{1}\right), \ldots,\left(Q_{k}, x_{k}\right)$ where, of course, existential (respectively, universal) quantifiers occur positively (negatively) in $A$. An argument similar to the one above establishes that $\Pi \subset \Pi_{1}^{0}$ and $\Lambda \subset \Sigma_{1}^{0}$. Thus $k$ applications of Lemma 4 show that there are terms $t_{1}, \ldots, t_{k}$ and a good $R$-proof $Q_{3}$ with endsequent

$$
\Pi \rightarrow D^{*}, \Lambda
$$

where $D^{*}$ is obtained from $D$ by replacing each unbounded quantifier $\left(Q_{i}, x_{i}\right)$ by the bounded quantifier $\left(Q_{i} x_{i} \leqslant t_{i}\right)$.

It is easy to modify $Q_{2}$ to obtain a proof $Q_{4}$ with endsequent $\Pi, D^{*} \rightarrow \Lambda$ so that $Q_{2}$ and $Q_{4}$ have the same number of collection inferences and so that $Q_{4}$ is also good.

Finally, we replace the subproofs $Q_{1}$ and $Q_{2}$ of $P^{*}$ by $Q_{3}$ and $Q_{4}$ and obtain a good proof with the same endsequent as $P$ and with fewer collection inferences than $P$. Applying the induction hypothesis yields an $R$-proof of $A$.

Theorem 1 is now proved, since if $R+B \Sigma_{1}^{0}$ proves the universal closure of a $\Sigma_{1}^{0}$-formula $A$ then, by Lemma 3 (cut elimination), there is a good proof of $A$, and hence, by Lemma 6, there is an $R$-proof of $A$.

The model-theoretic proof. We next present a second, model-theoretic proof of Theorem 1.

Let $R$ be any bounded, sufficient theory of arithmetic. If $M$ is a model of $R$, then the $\leqslant$ relation gives a linear ordering on $R$. A subset $I$ of $M$ is an initial segment of $M$ if and only if, $c \leqslant b$ implies $c \in I$ for all $b \in I$ and $c \in M$. We say that $I$ is closed under all operations if and only if $t(\vec{c}) \in I$, for every sequence $\vec{c}$ of elements of $I$ and every term $t$.

Definition. Let $M \vDash R$. The language $\mathscr{L}(M)$ is the language of $R$ enlarged to include a constant symbol for each element of $M$. If $A$ is any formula and $b \in M$, then $A \leqslant b$ is formed from $A$ by changing each unbounded quantifier $(\forall x)$ or $(\exists y)$ to the bounded quantifier $(\forall x \leqslant b)$ or $(\exists y \leqslant b)$. So $A^{\leqslant b}$ is an $\mathscr{L}(M)$-formula. When $z$ is a variable not occurring in $A, A^{\leqslant z}$ is defined similarly.

LEMma 7. If $M \vDash R, I$ is an initial segment of $M$ closed under all operations, $b \in I$, and $A$ is a $\Sigma_{1}^{0}$-formula in the language $\mathscr{L}(M)$, then

(a) $I \vDash R$,

(b) if $b<c$ and $M \vDash A^{\leqslant b}$, then $M \vDash A^{\leqslant c}$,

(c) if $M \vDash A \leqslant b$ and every constant symbol in $A$ denotes an element in $I$, then $I \vDash A$,

(d) if $I \vDash A$ and $c \in M \backslash I$, then $M \vDash A \leqslant c$.

Proof. (a) follows from the fact that $R$ is a bounded theory. (b)-(d) are easily proved by induction on the complexity of the $\Sigma_{1}^{0}$-formula $A$. 
We are now ready to prove Theorem 1 .

Proof of TheORem 1. Let $A\left(x_{1}, \ldots, x_{r}\right)$ be a $\Sigma_{1}^{0}$-formula with free variables as indicated such that $(\forall \vec{x}) A(\vec{x})$ is not a theorem of $R$. There exists a countable, recursively saturated model $M$ of $R+\neg A\left(c_{1}, \ldots, c_{r}\right)$, where $c_{1}, \ldots, c_{r}$ are now constant symbols. Since $M$ is countable and recursively saturated, it is also resplendent.

Let $m_{0}, m_{1}, m_{2}, \ldots$ enumerate the universe of $M$ and let $\theta_{0}, \theta_{1}, \theta_{2}, \ldots$ enumerate the $\Sigma_{1}^{0}$-formulae in the language $\mathscr{L}(M)$ with a single free variable $x$. So $\boldsymbol{\theta}_{i}=\boldsymbol{\theta}_{i}\left(x, \vec{n}_{i}\right)$, where $\vec{n}_{i}$ is a vector of elements of $M$. We shall define a sequence of models $M_{0}, M_{1}, M_{2}, \ldots$ and a sequence of elements $a_{0}, a_{1}, a_{2}, \ldots$ so that

(1) $M_{i+1}$ is an initial segment of $M_{i}$ closed under all operations;

(2) $a_{i} \in M_{i}$;

(3) $a_{i+1} \geqslant a_{i}$; and

(4) each $M_{i}$ is recursively saturated and hence resplendent.

Begin by defining $a_{0}=\max \left(c_{1}, \ldots, c_{r}\right)$ and $M_{0}=M$.

Now suppose $a_{k}$ and $M_{k}$ have been defined. Let $i=\beta(1, k)$ and $j=\beta(2, k)$, where $\beta$ is the Godel sequence coding function. Consider the element $m_{i} \in M$ and the formula $\theta_{j}\left(x, \vec{n}_{j}\right)$. There are three cases:

Case (1). $m_{i} \notin M_{k}$ or $\vec{n}_{j} \notin M_{k}$ or $M_{k} \vDash \neg\left(\forall x \leqslant m_{i}\right) \theta_{j}(x)$. Set $M_{k+1}=M_{k}$ and $a_{k+1}=a_{k}$.

Case (2). $M_{k} \vDash\left(\forall x \leqslant m_{i}\right) \theta_{j}(x)$ and there is a $b \in M_{k}$ such that

$$
M_{k} \vDash\left(\forall x \leqslant m_{i}\right) \theta_{j} \leqslant b(x) .
$$

In this case, let $M_{k+1}=M_{k}$ and let $a_{k+1}$ be the maximum of $a_{k}$ and $b$.

Case (3). Neither of the above cases holds. Let $I$ be the initial segment of $M_{k}$ defined by

$$
I=D\left\{b \in M_{k}: b \leqslant t\left(a_{k}\right) \text { for some term } t\right\} .
$$

By the recursive saturation of $M_{k} . I \neq M_{k}$ (except in the degenerate case where $M_{k}$ has a maximum element). Also, $I$ is closed under all operations and $a_{k} \in I$. Hence

$M_{k} \vDash(\exists$ predicate $Q)\left(Q\right.$ is a proper initial segment, containing $a_{k}$ and is closed under all operations).

Namely, choose $Q$ to be $I$. By the resplendency of $M_{k}$, there is a (different) predicate $Q_{k}$ so that all of the above properties hold and so that the expanded structure $\left(M_{k}, Q_{k}, \ldots\right)$ is resplendent.

By Lemma 7(d) and since Case 2 did not hold.

$$
Q_{k} \vDash \neg\left(\forall x \leqslant m_{i}\right) \theta_{j}\left(x, \vec{n}_{j}\right) .
$$

Now define $a_{k+1}=a_{k}$ and $M_{k+1}=Q_{k}$.

This completes the definition of $M_{0}, M_{1}, M_{2}, \ldots$.

Let $M_{\omega}$ be defined by

$$
M_{\omega}=\bigcap_{i=0}^{\infty} M_{i}
$$


We claim that $M_{\omega} \vDash R+B \Sigma_{1}^{0}$ and yet $M_{\omega} \vDash \neg A(\vec{c})$. This suffices to prove Theorem 1 since it implies that $R+B \Sigma_{1}^{0}$ does not prove $(\forall \vec{x}) A(\vec{x})$. Since $M_{\omega}$ is an initial segment of $M$ and $M_{\omega}$ is closed under all operations, Lemma 7(a) implies $M_{\omega} \vDash R$. In addition since $A \in \Sigma_{1}^{0}, M_{\omega} \vDash \neg A(\vec{c})$.

An arbitrary instance of a $B \Sigma_{1}^{0}$-collection axiom over $M_{\omega}$ is of the form

$$
\left(\forall x \leqslant m_{i}\right)(\exists y) \varphi(x, y, \vec{n}) \rightarrow(\exists z)\left(\forall x \leqslant m_{i}\right)(\exists y \leqslant z) \varphi(x, y, \vec{n}),
$$

where $m_{i}, \vec{n} \in M_{\omega}$ and $\varphi \in \Sigma_{1}^{0}$. Let $j$ be such that $\theta_{j}\left(x, \vec{n}_{j}\right)$ is $(\exists y) \varphi(x, y, \vec{n})$. It will suffice to show that

$$
M_{\omega} \vDash\left(\forall x \leqslant m_{i}\right) \theta_{j}\left(x, \vec{n}_{j}\right) \rightarrow(\exists z)\left(\forall x \leqslant m_{i}\right) \theta_{j} \leqslant z\left(x, \vec{n}_{j}\right) .
$$

Let $k=\langle i, j\rangle$, so $\beta(1, k)=i$ and $\beta(2, k)=j$. Examine the way in which $M_{k+1}$ was defined. If $M_{k+1}$ was defined by Case (1) or Case (3), then $\left(\forall x \leqslant m_{i}\right) \theta_{j}\left(x, \vec{n}_{j}\right)$ is false in $M_{k+1}$. Since $M_{\omega}$ is an initial segment of $M_{k+1}$ closed under all operations and since $\theta_{j} \in \Sigma_{1}^{0}$,

$$
M_{\omega} \vDash \neg\left(\forall x \leqslant m_{i}\right) \theta_{j}\left(x, \vec{n}_{j}\right) .
$$

If $M_{k+1}$ was defined via Case (2), then

$$
M_{k+1} \vDash\left(\forall x \leqslant m_{i}\right) \theta_{j} \leqslant a_{k+1}\left(x, \vec{n}_{j}\right) .
$$

Since $a_{k+1} \in M_{\omega}$ and $\theta_{j} \leqslant a_{k+1}$ is bounded.

$$
M_{\omega} \vDash(\exists z)\left(\forall x \leqslant m_{i}\right) \theta_{j} \leqslant z\left(x, \vec{n}_{j}\right) .
$$

\section{REFERENCES}

1. S. R. Buss, Bounded arithmetic, Bibliopolis, Naples, 1986. Revision of Ph. D. dissertation, Princeton University, 1985.

2. R. Parikh, Existence and feasibility in arithmetic, J. Symbolic Logic 36 (1971), 494-508.

3. J. B. Paris, Some conservation results for fragments of arithmetic, Model Theory and Arithmetic, Lecture Notes in Math., vol. 890, Springer-Verlag, Berlin and New York, 1980, pp. 251-262.

4. J. B. Paris and L. A. S. Kirby, $\Sigma_{n}$-collection schemes in arithmetic, Logic Colloquium ' 77 , North-Holland, Amsterdam, 1978, pp. 199-209.

5. J. P. Ressayre, A conservation result for systems of bounded arithmetic, handwritten notes, 1985.

6. G. Takeuti, Proof theory, North-Holland, Amsterdam, 1975.

Mathematical Sciences Research InStitute, Berkeley, California 94720

Current address: Department of Mathematics, University of California, Berkeley, California 94720 